\title{
Skin tears. A quantitative study on the phenomenon and proposal for a prevention and management procedure
}

\author{
Giulia Candeloro \\ ASST Grande Ospedale Metropolitano Niguarda, Milan, Italy
}

\begin{abstract}
The study aims to understand whether healthcare professionals, nurses and hospital aides know skin tears and how to recognize, prevent and manage them. In addition, it seeks to understand if there is a need for an instrument that supports operators in recognizing these lesions. The last aim is to propose a procedure for the prevention and management of skin tears. Existing literature on the issue has been reviewed and a quantitative study was conducted on skin tears by administering a questionnaire of 18 questions to nurses and hospital aides. Nurses and hospital aides are confused as regards the management of skin tears and need a tool to guide them in healthcare. A procedure for the prevention and management of skin tears is proposed.
\end{abstract}

\section{INTRODUCTION}

According to Payne and Martin, ${ }^{1}$ Carville et al. ${ }^{2}$ and LeBlanc et al., ${ }^{3}$ skin tears are a lesion caused by shear forces, friction and/or blunt bodies, causing the separation of skin layers. They may be: i) Partial thickness: with separation of the epidermis from the dermis; ii) Full thickness: with the separation of the epidermis and dermis from the underlying structures.

Those most affected are people: i) Of very young or old age (infants and the elderly); ii) In critical conditions or who are clinically compromised; iii) Requiring assistance for their personal care (ICU, major surgery, or traumatology patients).

Correspondence: Giulia Candeloro, ASST Grande Ospedale Metropolitano Niguarda, Piazza dell'Ospedale Maggiore, 320162 Milan, Italy.

Tel.: 0264441.

E-mail: giulia.candeloro@ospedaleniguarda.it

Key words: skin tears, quantative study, prevention and management.

Conflict of interest: the author declares no potential conflict of interest.

Received for publication: 28 August 2017.

Revision received: 28 August 2017.

Accepted for publication: 10 October 2017.

This work is licensed under a Creative Commons Attribution NonCommercial 4.0 License (CC BY-NC 4.0).

(C) Copyright G. Candeloro, 2017

Licensee PAGEPress, Italy

Italian Journal of Wound Care 2017; 1(2):61-66

doi:10.4081/ijwc.2017.13

\section{Risk factors}

The factors that may contribute to the onset of a skin tear are: age; sex (female); race (Caucasian); dehydration; malnutrition; cognitive impairment; altered mobility; impaired vision; neuropathies; long-term therapy with corticosteroids; prior history of skin tear; stiff limbs and spasticity; blood samples; combination therapy; presence of bruising; dependence in activities of daily living (ADL); use of assistive devices; taking socks on and off; removing plasters and dressings; vascular issues; heart issues; lung issues; transfers and falls; prosthetic devices; continence/incontinence; skin cleansers.

\section{Prevention}

The literature ${ }^{4,5}$ suggests that it is possible to reduce the incidence of skin tears by at least $50 \%$ through the implementation of specific protocols and staff training, which is the crucial aspect for the prevention of skin tears: all caregivers should know but especially apply some simple and common strategies. The best known strategies are LeBlanc et al. ${ }^{6}$

\section{The classification of skin tears}

Today, there is no universally accepted and properly validated classification system for the assessment of skin tears in the literature, although the literature often shows an increasing use of the classification made in 1980 by Payne and Martin, later revised in 1993 (Table 1). ${ }^{7}$

Another classification that is used for guidance in the classification of skin tears is the one developed in 2006 in Australia: the STAR (Skin Tear Audit Research) Classification System ${ }^{2}$ (Table 2). ${ }^{7}$

These two classification systems, though apparently very similar, distinguish skin tears based on different factors: the first classification (Payne and Martin) is based on 
the extent of tissue loss; the second (STAR Classification System), instead, on the quality of the edges and skin flap.

At any rate, regardless of the system used, it is not correct - though this is often the case - to classify skin tears using the staging systems for pressure ulcers. ${ }^{1,5}$

\section{Treatment}

The first step to develop a treatment plan for skin tears is to complete the wound assessment. ${ }^{8}$

The steps in treating a skin tear recognized in the literature are: i) Cleansing: removing bacteria, debris and/or any necrotic tissue present; ii) Healing in a damp environment; iii) Selection of dressings: Following the indications of $\mathrm{RNAO}^{9}$ on the use of a good dressing. In the case of skin tears, the literature features several studies and indications. Sutton and Pritty ${ }^{10}$ on the use of steri strips instead of the sutures or staples due to fragile skin; Nazarko ${ }^{11}$ on the application of calcium alginate to control post-injury bleeding; LeBlanc and Christensen ${ }^{12}$ on the use of transparent absorbent acrylic dressings. iv) Management of the cutaneous flap; v) Management of oedema; vi) Pain management; vii) Management of infection: with the prevention and, if present, the application of topical antimicrobials and/or systemic antibiotics; viii) Dressings to avoid: hydro- colloids and transparent films. They may cause epidermal stripping and interfere with the healing of skin tears, if they are not removed accordingly.

\section{Objectives}

The study set the following objectives: i) Understanding whether there are patients at risk of skin tear in the studied healthcare settings; ii) Understanding whether nurses and hospital aides know how to correctly manage skin tears; iii) Understanding whether, for respondents, there is the need for a specific instrument to prevent and deal with this type of lesion; iv) Creating a proposed procedure for the prevention and management of skin tears.

\section{MATERIALS AND METHODS}

The quantitative research started in February 2014, with the development of an 18-question questionnaire. The questionnaire was administered both to nurses and hospital aides.

The operating units to which the test was distributed were those present at the hospital and the local health care clinics.

Specifically: i) Intensive Care Unit; ii) Emergency Care Unit; iii) Surgery and Urology Unit; iv) Or-

Table 1. Payne and Martin classification. Reproduced with permission from Stephen Haynes et al. (2011). ${ }^{7}$

CATEGORY IA Linear skin tear with separated epidermis and dermis, without tissue loss.

CATEGORY IB The epidermal flap completely covers the dermis within one millimetre of the wound margin.

CATEGORY IIA Scant tissue loss $<25 \%$ of the lost epidermal flap.
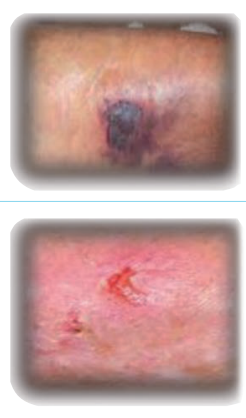

CATEGORY IIB Tissue loss $>25 \%$ of the lost epidermal flap.

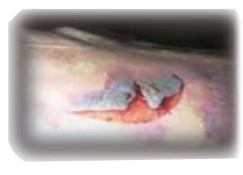

CATEGORY III Complete tissue loss. The epidermal flap is absent.

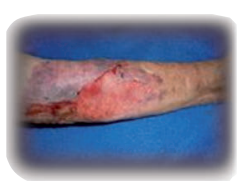


thopaedics Unit; v) Psychiatry Unit; vi) Medicine B Unit; vii) Medicine $C$ Unit; viii) Bronchopneumology Unit; ix) Neurology Unit; x) Cardiology Unit; xi) Integrated Home Care (ADI); xii) Oncology Home Care (ADI); xiii) Nursing Home Care (RSA) No. 1; xiv) Nursing Home Care (RSA) No. 2.

The Units which were excluded from the study were: i) All the outpatient clinics; ii) Gynaecology and Obstetrics Unit; iii) Paediatrics and Neonatal Pathology Unit.

The paediatrics/neonatal pathology and gynaecology units, though having a patient at risk of skin tears (as reported by scientific studies), were excluded from the quantitative research, as we chose to place greater attention on the elderly patient who belongs to an increasingly growing population present at the hospital and local clinics.

After processing the survey instrument, the questionnaire was administered. The tests were distributed over two days. Recipients of the study were given about a month of time to complete the test.

\section{RESULTS}

One month after the distribution of the 239 administered tests, 113 were completed and 126 were not (Figure 1).
Specifically, $89 \%$ were filled out by nurses and $11 \%$ by hospital aides (Figure 2).

The analysis of the results was done for all operating

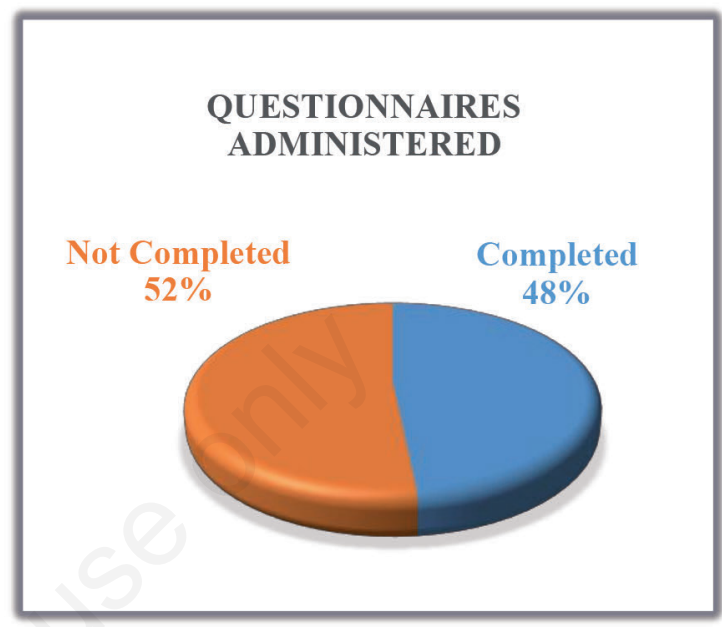

Figure 1. Percentual questionnaire compiled and not compiled by the study group.

Table 2. STAR classification system (2007). Reproduced with permission from Stephen Haynes et al. (2011). ${ }^{7}$

CATEGORY IA A skin tear where the edges can be realigned to the normal anatomical position and the skin or flap colour is not pale, dusky or darkened.

CATEGORY IB A skin tear where the edges can be realigned to the normal anatomical position and the skin or flap colour is pale, dusky or darkened.

CATEGORY IIA A skin tear where the edges cannot be realigned to the normal anatomical position and the skin or flap colour is not pale, dusky or darkened.

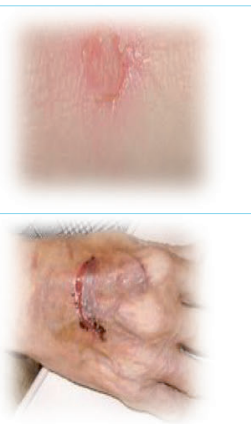

CATEGORY IIB A skin tear where the edges cannot be realigned to the normal anatomical position and the skin or flap colour is pale, dusky or darkened.

CATEGORY III A skin tear where the skin flap is completely absent. 
units involved in the study. The overall view of the collected data follows below.

The question shows the degree of participation of the professionals involved in the study: $89 \%$ of nurses and $11 \%$ of hospital aides. While the participation of hospital aides was low, that of the nursing staff was: good, as regards the ICU, Emergency, Neurology and two home care units (A.D.I. and A.D.O.); fair for the surgical areas (Surgery Urology and Orthopaedics) and Psychiatry and, finally, minimal as regards the hospital and local medical areas (Medicine B and C, Bronchopneumology, Cardiology, Nursing Home Care 1 and 2), where the probability of having patients with this type of lesion is actually much higher.

Moderate interest ( $89 \%$ of respondents) in the prevention and management of cutaneous alterations of the upper and/or lower limbs and therefore also in skin tears.

From question 3 to 9 (included), the collected data show that the probability of having patients with skin tears is very high. In fact: $94 \%$ is affected by multiple pathologies; in $92 \%$ of cases, normal cutaneous trophism is altered (with a frequency of $20 \%$ ); about $90 \%$ of patients has a nutritional deficit, although the frequency of its being identified is still low in most cases (about 20\%); around $88 \%$ shows states of psychomotor agitation (especially the patients in critical areas, Cardiology, Neurology, Bronchopneumology, Medicine B and C, Orthopaedics and Nursing Home Care 1); about $61 \%$ of patients is aged between 70 and 85 years.

Then, question No.10 shows that skin tears are identified often by $56 \%$ of respondents and seldom for the remaining 41\% (Figure 3).

Question No. 11 shows that the frequency to document this type of lesion varies. In question No. $12,75 \%$ of nurses and hospital aides admit to treat skin tears following the hospital protocol (i.e., a standardised indication for the prevention and management of pressure ulcers), while only $23 \%$ of respondents stated to act according to their professional knowledge (Figures 4 and 5).

About $85 \%$ of the respondents identify prevention as a fundamental process applicable in their work routine in order to prevent the onset of skin tears. Degeneration, instead, is considered a rare occurrence.

In question No.17, almost all the nurses and aides (97\%) agree in saying that the presence of any skin lesion, even of an acute wound like skin tear, if present, can negatively affect the mental and physical well-being of a patient.

Finally, in the last question $94 \%$ of nurses and aides stated that they need a more specific indication for the prevention and treatment of skin tears (Figure 6).

Therefore, concluding this analysis, we can say that the aims at the beginning of this quantitative study have all been achieved: i) The relevance of patients at risk of skin tears in the clinical and local facilities of the hospital examined; ii) The presence of widespread confusion about the management of skin tears, thus confirming LeBlanc's findings in the literature; ${ }^{3}$ iii) The need to have a tool that helps healthcare professionals to prevent and manage skin tears.

\section{Limits of the study}

The first limit encountered was in the use of the term skin tear in question No. 10. It was decided not to use this term, but synonyms, to avoid possible consultations of the web that would alter the veracity of the test.

The second limit was the participation of the candidates in the study: more than half of the tests were not completed.

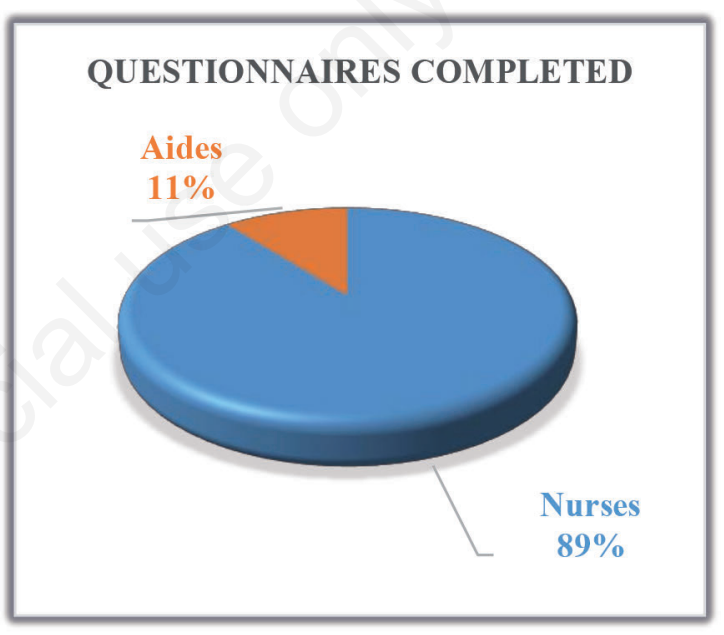

Figure 2. Percentage of nurses and oss who participated in the study.

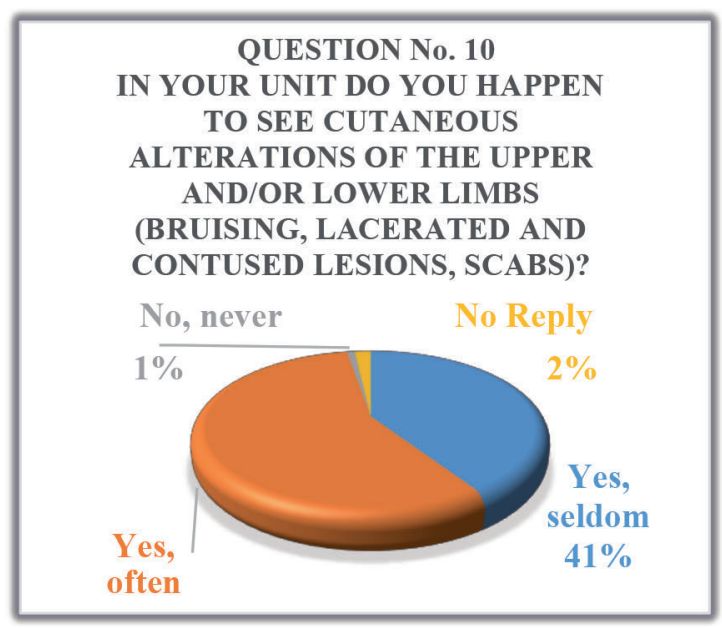

Figure 3. Frequency of detection of skin tears by the study group. 


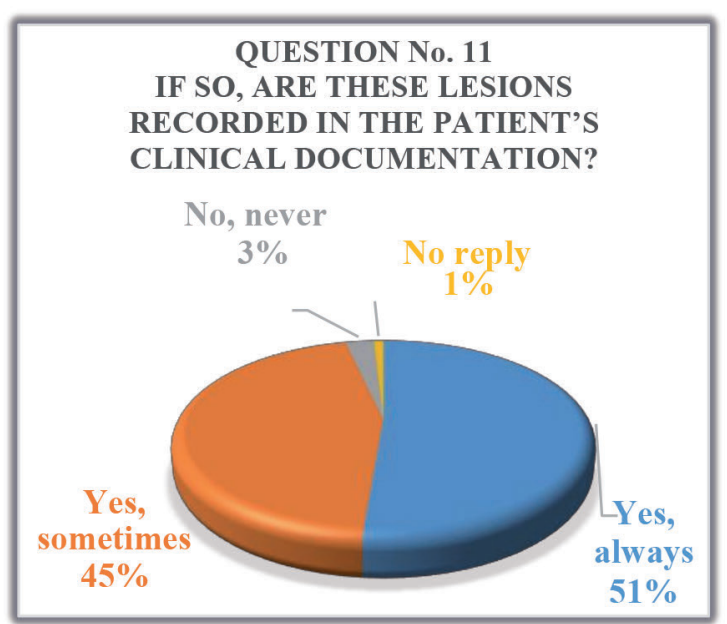

Figure 4. Frequency of skin tears recording in clinical documentation.

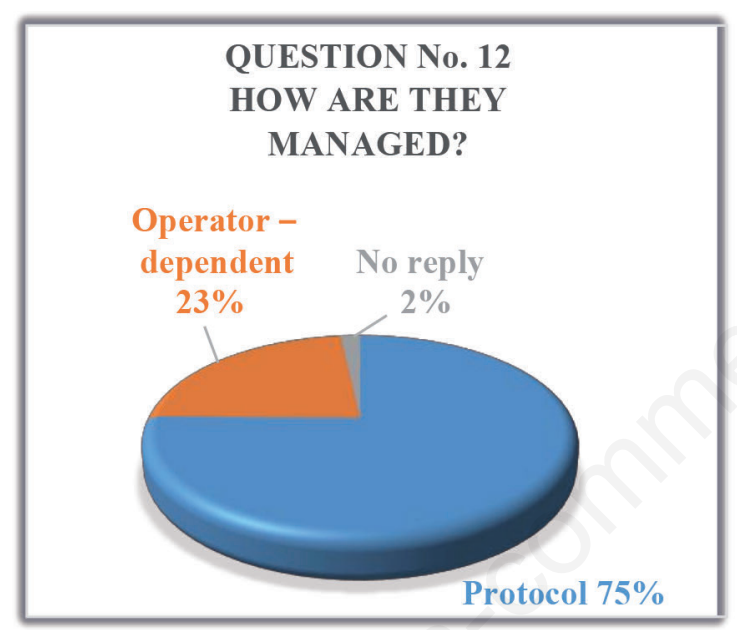

Figure 5. Percentage on how to treat skin tears.

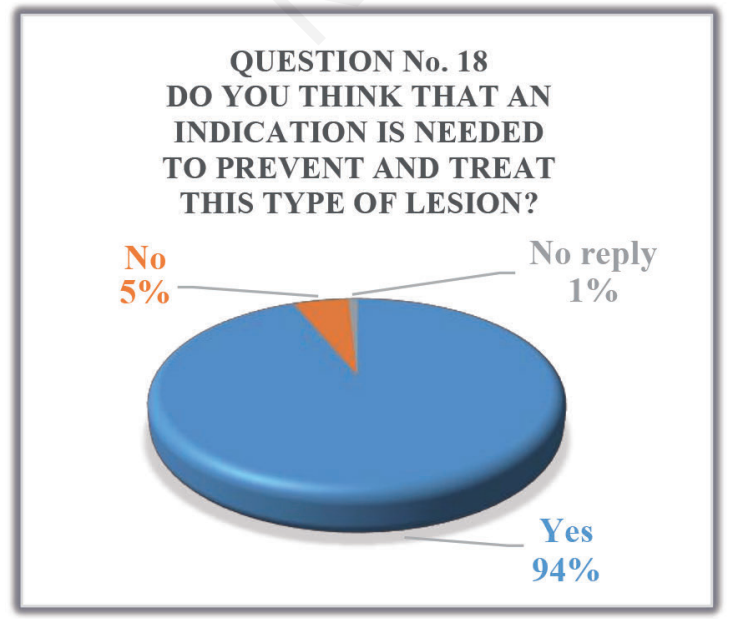

Figure 6. Percentage of those who consider it necessary an indication for the prevention and management of skin tears.
Finally, the confusion found with regard to the difference between prevention and treatment of a pressure ulcer and a skin tear.

\section{CONCLUSIONS}

At the end of this quantitative study, it can be concluded that skin tears are known but not fully understood acute lesions where there is still much to discover and improve, especially as regards the clinical management and training of healthcare personnel.

Hence the idea of wanting to propose a procedure that can help, through the use of certain instruments (Figures $7^{7}, 8^{3,5}$ ) to prevent, recognize, manage and document this type of acute wound.

The procedure consists of 6 indicators and assigns different responsibilities to nurses, hospital aids, nutritionists, general practitioners and to caregivers and/or patients (depending on the patient's level degree of self-sufficiency).

We recommend 4 scales, already known in the literature, to assess risk: MMSE, ADL, MNA, VRS, NRS and PBS.

As regards the treatment of skin tears, reference is made to the current state of knowledge in the literature and in clinical practice.

Finally, a draft sheet for the assessment and management of the wound has been created to gather information on healthcare professionals' work (Figures 9, 10).

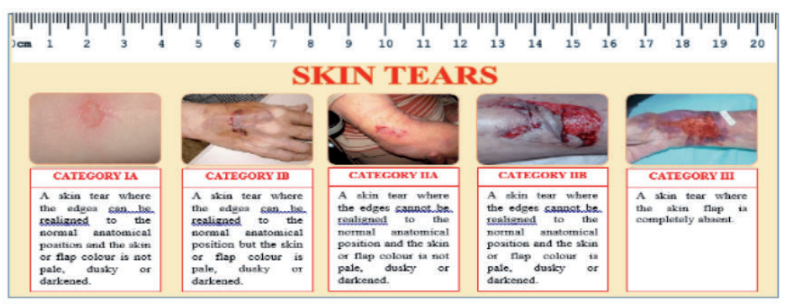

Figure 7. Skin tear classification tool. Reproduced with permission from Stephen Haynes et al. (2011). ${ }^{7}$

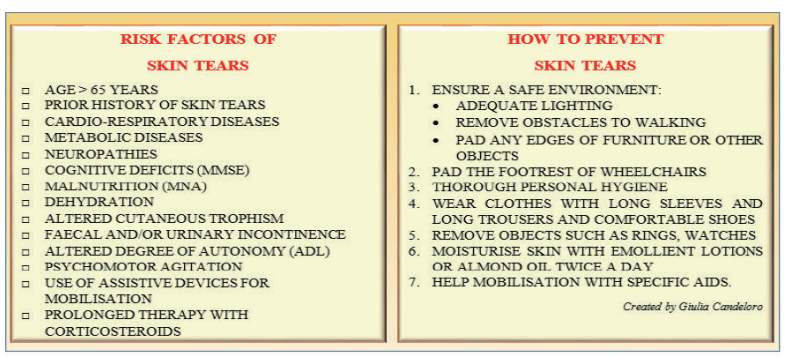

Figure 8. Skin tear prevention tool. Reproduced with permission from LeBlanc et al. (2008). ${ }^{3}$ Reproduced with permission from Caula et al. (2011). ${ }^{5}$ 


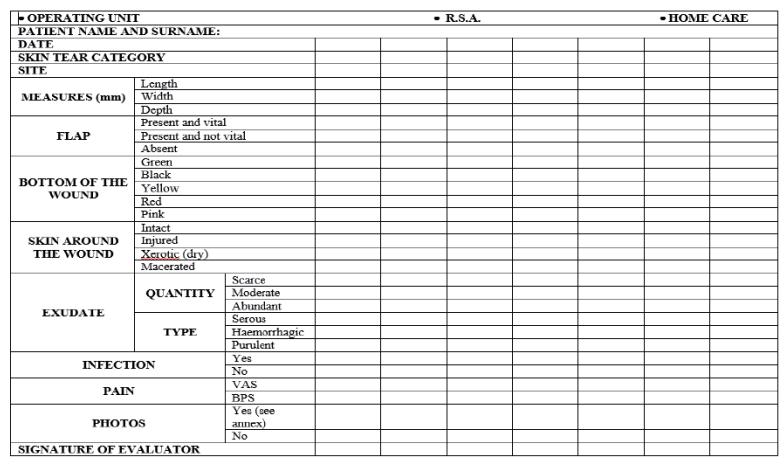

Figure 9. Skin tear evaluation sheet.

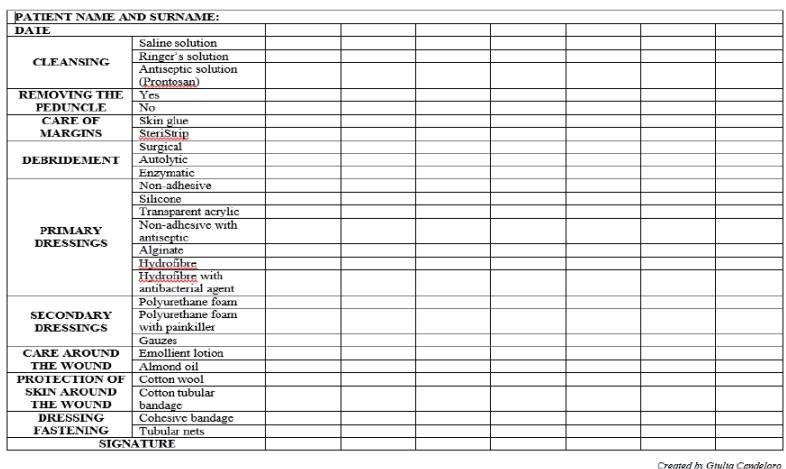

Figure 10. Skin tear dressing table.

\section{REFERENCES}

1. Payne RL, Martin ML. The epidemiology and management of skin tears in older adults. Ostomy Wound Manage 1990;26:26-37.

2. Carville K, Lewin G, Newall N, et al. STAR: a consensus for skin tear classification. Prim Intent 2007;15:18-28.

3. LeBlanc K, Christensen D, Orstead H, Keast D. Best practice recommendations for the prevention and treatment of skin tears. Wound Care Canada 2008;1:14-30.

4. Ratliff CR, Fletcher KR. Skin Tears: a review of the evidence to support prevention and treatment. Ostomy Wound Manage 2007;53:32-40.

5. Caula C, Apostoli A. Cura e assistenza al paziente con ferite acute e ulcere croniche. Manuale per l'infermiere. Sant'Arcangelo di Romagna: Maggioli; 2011. pp 385-389.

6. LeBlanc K, Baranoski S. Skin Tears: state of the science: consensus statement for the prevention, prediction, assessment, and treatment of skin tears. Adv Skin Wound Care 2011;24:2-15.

7. Stephen Haynes J, Carville K: Skin tears Made easy, Wound International, 2011;2:1-6.

8. Le Blanc K, Baranosky S. Prevention and management of Skin Tears, Adv Skin Wound Care 2009;22:325-32.

9. Registered Nurses' Association Of Ontario (RNAO): Nursing best practice guideline: risk assessment and preention of pressure ulcers. Toronto 2005.

10. Sutton R, Pritty P. Use of sutures or adhesive tapes for primary closure of pre-tibial lacerations. Br Med J (Clin Res Ed) $1985 ; 290: 1627$.

11. Nazarko L. Preventing and treating skin tears, Nurs Resident Care 2005;7:549-50.

12. LeBlanc K, Christensenn D. An approach to managing skin tears in the elderly population: a case series. Poster Presentation presented at the Canadian Association of Wound Care Annual Conference. Montreal, QC; 2005. 